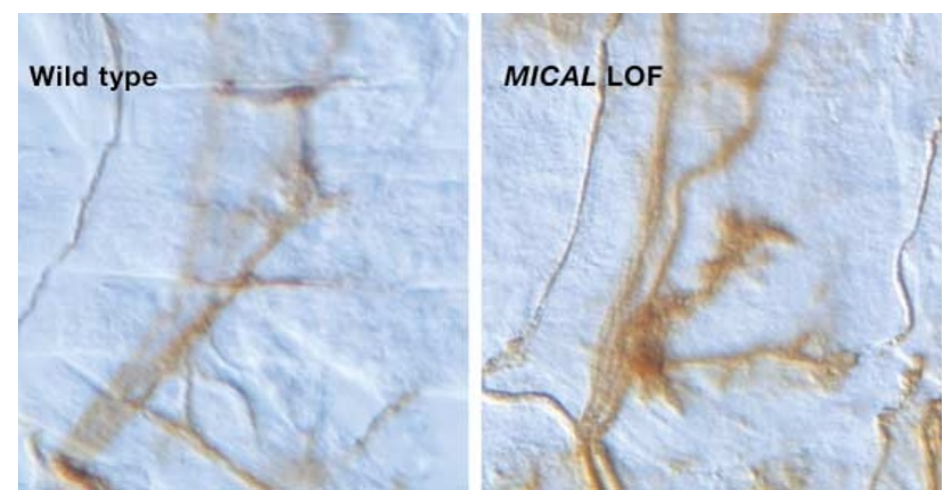

Axon-guidance defects in a Drosophila MICAL loss-of-function (LOF) mutant. Courtesy of Alex Kolodkin, Johns Hopkins School of Medicine, Baltimore, USA.

AXON GUIDANCE

\section{MICAL — a missing link}

The semaphorins are important axon-guidance molecules in invertebrates and vertebrates, and they can cause attraction or repulsion. The repulsive response is mediated by the plexin family of receptors, but little is known about how the plexins signal to the cytoskeleton to induce growth cone collapse. Now, a new study implicates a family of oxidoreductases, the MICALs, as a missing link in this pathway.

The identification of the vertebrate MICAL-1 (molecule interacting with CasL) was reported earlier this year, and now Terman et al. describe the isolation of a Drosophila counterpart, and of vertebrate MICAL-2 and -3. MICALs are large cytosolic proteins that consist of several domains. In the Drosophila embryo, MICAL shows a similar expression pattern to Plexin A (PlexA) on central nervous system and motor axons, and the authors showed that it interacts physically with PlexA. MICAL also contains an actin-binding domain and an intermediate-filament-binding region, so it could provide a direct link between the plexins and the cytoskeleton. Vertebrate MICAL- 1 interacts with CasL, and as Cas-family proteins interact with signalling proteins that influence neuronal morphology, MICAL could link plexins to cytoskeletal regulators through Cas proteins.

Terman et al. looked for evidence that MICAL acts in the same pathway as Semaphorin 1a (Sema-1a) and PlexA. They found that MICAL lossof-function caused the same axonguidance and defasciculation defects as were seen in PlexA and Sema-1a mutants, and overexpression of MICAL had the same effect as overexpression of PlexA. Further evidence for a common pathway came from embryos that were doubly heterozygous for mutations in MICAL and PlexA or Sema-1a, which showed similar axon-guidance defects to Sema-1a;PlexA double heterozygotes. Which domains in MICAL are important for its function in semaphorin-mediated repulsion? The amino terminus is highly conserved between species, and this region contains a flavoprotein monooxygenase domain, which catalyses an oxidation-reduction (redox) reaction that results in the insertion of an oxygen atom into a substrate. Alterating key amino-acid residues within this domain that are conserved within this enzyme class and are required for their activity compromises MICAL function in motor axons in vivo. In an in vitro rat growth cone assay, Terman et al. showed that semaphorinmediated axonal repulsion can be inhibited by selective flavoprotein monooxygenase inhibitors.

So, these findings not only tell us more about the signalling pathway that underlies semaphorin-mediated repulsion, but in combination with recent data that implicate 12/15lipoxygenase in semaphorin signalling, they also provide support for the involvement of redox reactions in this pathway. Oxidation of actin is known to promote its depolymerization, so it will be interesting to see whether redox reactions are a common feature of repulsive neuronal guidance.

Heather Wood

\section{(2) References and links} ORIGINAL RESEARCH PAPER Terman, J. R. et al. MICALs, a family of conserved flavoprotein oxidoreductases, function in plexin-mediated axidoreductases, function in plexin-mediated FURTHER READING Finkel, T. Redox-dependent signal transduction. FEBS Lett. 476, 52-54 (2000) | Tamagnone, L. \& Comoglio, P. M. signalling by semaphorin receptors: cell guidance and beyond. Trends Cell Biol. 10, 377-383 (2000)

\section{PRION PROTEINS}

\section{Protector prions}

Two papers published in EMBO Journal hint at a neuroprotective function for the cellular prion protein, $\operatorname{PrP}^{\mathrm{C}}$. Mutations in this protein, or changes in conformation induced by the infective prion protein $\mathrm{PrP}^{\mathrm{sc}}$, can cause spongiform encephalopathies such as scrapie, bovine spongiform encephalopathy and CreutzfeldtJakob disease.

However, the function of $\operatorname{PrP}^{\mathrm{C}}$ is still a mystery. It is not even clear whether the neurodegeneration that is associated with spongiform encephalopathies is caused by a loss of the normal function of $\operatorname{PrP}^{\mathrm{C}}$ or by a gain of toxic function by $\operatorname{PrP}^{\mathrm{Sc}}$. The apparent absence of a phenotype in prion-knockout mice argued for the latter, but evidence is accumulating that cellular prions might have a neuroprotective role, the loss of which could cause cell death in prion diseases.

The two new papers both come from the same research group. One, by Zanata et al., shows that the cellular prion binds to a heatshock-related protein, stress-inducible protein 1 (STI1), and that the interaction between these two proteins at the cell surface can rescue cultured retinal cells from apoptosis induced by treatment with anisomycin. A functional role for this interaction is supported by the finding of both proteins on neuronal cell surfaces in the central nervous system. The second paper, by Chiarini et al., shows that binding of $\operatorname{PrP}^{\mathrm{C}}$ by a peptide that recognizes the STI1-binding site (known as $\operatorname{PrR}$ ) also protects neurons against apoptosis in vitro. It goes further to demonstrate that this protection depends on an increase in the levels of cyclic AMP that activates protein kinase $\mathrm{A}$.

Many questions are outstanding. For example, it is unclear how the binding of $\operatorname{PrP}^{\mathrm{C}}$ at the outer membrane activates adenylyl cyclase, which is normally regulated by $G$ proteins on the inner membrane. And it remains to be shown that the neuroprotective effect of PrP $\mathrm{P}^{\mathrm{C}} \mathrm{STI1}$ binding is physiologically relevant in vivo, or that it is disrupted by infection with $\operatorname{PrP}^{\mathrm{sc}}$. But a fuller understanding of signalling and neuroprotection by $\operatorname{PrP}^{\mathrm{C}}$ should help us to understand the pathogenesis of prion disorders as well as the underlying cell biology of these proteins.

\section{(2) References and links}

Rachel Jones

ORIGINAL RESEARCH PAPER Zanata, S. M. etal. Stress-inducible protein 1 is a cell surface ligand for cellular prion that triggers neuroprotection. EMBO J. 21, 3307-3316 (2002) Chiarini, L. B. et al. Cellular prion protein transduces neuroprotective signals. EMBO J. 21 $3317-3326(2002)$

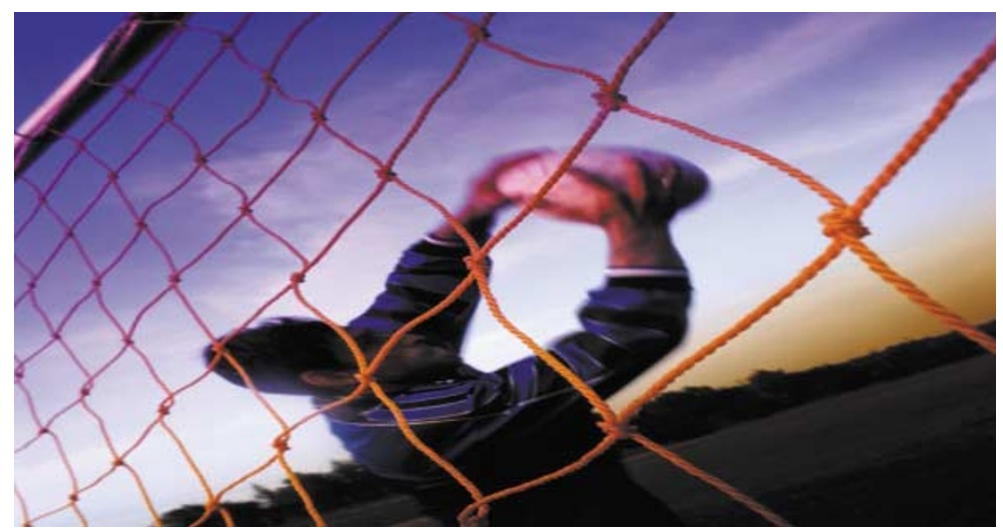

